\title{
Analysis and Prevention of Animal-Caused Faults in Power Distribution Systems
}

\author{
Mo-yuen Chow \\ IEEE Senior Member \\ Dept. of Electrical and Comp. Engineering
North Carolina State University \\ Raleigh, NC 27695-7911
}

\section{Abstract}

The reliability, security and quality of power systems are significantly affected by distribution faults. One of the current trends concerning distribution faults is the implementation of more preventive measures instead of reactive measures. In other words, we would like to prevent faults from happening rather than to restore the system after faults occur. Among the different categories of power distribution faults, animal-caused faults are probably the ones that can be prevented most easily and effectively if appropriate control actions are taken. This paper will discuss and analyze animal-caused faults in power distribution systems. The results of an animal fault prevention technique that can effectively reduce these faults will also be presented.

Keywords : Power distribution systems, distribution system faults, animal faults, preventive maintenance, control action fault prevention.

\section{Introduction}

Electrical service to distribution customers can be affected by a variety of events including animals, winds, trees, lightning, equipment failure, and others. Any of these events can cause a system disturbance. Power system devices are designed to minimize the effects of these disturbances to the power system and its customers. The process of clearing a disturbance or the disturbance itself can result in the customer experiencing a complete loss of power or a decrease in the supplied voltage. Reducing the number of disturbances in the distribution lines is the primary focus of reliability programs of power utility companies. In the past, several papers have discussed about the impact of distribution faults on the reliability, economic and safety issues of distribution system operations [1-3,6-10].

At Duke Power Company, with distribution systems in North Carolina and South Carolina, there are 1,787 circuits, 1,694,557 customers, 265,472 fuse locations, 4,295 recloser locations, 501,073 overhead transformers, 91,916 underground transformers, and 55,436 primary line miles (including overhead and underground cables). There are, on the average each year, 40,000 permanent distribution faults (which cause outages),

94 SM 470-5 PWRD A paper recommended and approved by the IEEE Transmission and Distribution Committee of the IEEE Power Engineering Society for presentation at the IEEE/PES 1994 Summer Meeting, San Francisco, CA, July 24 - 28, 1994. Manuscript submitted July 26, 1993; made available for printing May 3, 1994.
Leroy S. Taylor

IEEE Member

\author{
Power Delivery Engineering Services \\ Duke Power Company \\ Charlotte, NC 28201-1010
}

30,000 major temporary faults that trip the main circuit breakers, and 75,000 minor temporary faults that trip line breakers or reclosers (which do not cause outages). Likewise, many other utility companies also experience a large number of distribution faults in their systems. In the area of power quality and reliability of distribution circuits, there is significant emphasis on techniques and technologies that can be used to react to utility system faults. One of the trends concerning distribution faults is the implementation of more preventive measures instead of reactive measures. In other words, we would like to prevent faults from happening rather than to restore the system after faults happen. The prevention of distribution faults is a difficult task, not only because the fault causes are affected by many different variables, but also because distribution faults have their own local properties [3]. Different geographical locations require different methods of fault prevention.

One approach to prevent distribution faults is to modify and improve the design of existing distribution systems based on the knowledge about previous distribution faults occurring in the system. For example, if in the past a distribution system has experienced many faults caused by animals that climb the distribution poles and short the phase to ground wires, then based on this knowledge we can design a system that gives a larger clearance between the phase to ground wires to avoid this type of problem. However, in most cases, once the distribution systems have been installed and are in operation, it is too costly to replace them with newer and better designed systems. Also, environmental conditions may incur different types of faults unforeseen at the time of the system design. On the other hand, if a system experiences frequent fault occurrences, then rather than constantly restoring the system after faults occur or installing new systems, it may be more efficient and cost effective to determine and eliminate the fault sources through control action, so that similar faults can be prevented from occurring at that site in the future. For instance, if many faults are identified as being caused by animals that short the phase to ground wires on a distribution transformer, then we can install an animal guard on the transformer to prevent future faults of this type.

Fig. 1 shows a dead squirrel on top of a transformer. The top of the transformer is at ground or neutral potential and the top of the bushing is 7,200 Volts with respect to neutral. The top of this transformer used to have an insulated covering, but the paint did not serve to insulate the cover. Most animal outages create clouds of ionized, conductive gases allowing a maximum fault limited only by the impedance of the system. Such a fault can create a momentary outage, voltage sag, or possibly a sustained (long duration) outage on the distribution circuit. Many utility customers can be affected by one animal 
outage. An animal guard should have been installed around the top of the transformer high voltage bushing to prevent a phase to ground fault from occurring. This type of fault prevention method is a Control Action Fault Prevention technique. Trimming trees to prevent tree faults is another example of a Control Action Fault Prevention technique.

Dead squirrel body that was electrocuted by cauping a phase to ground fault on the transfolmer bushings.

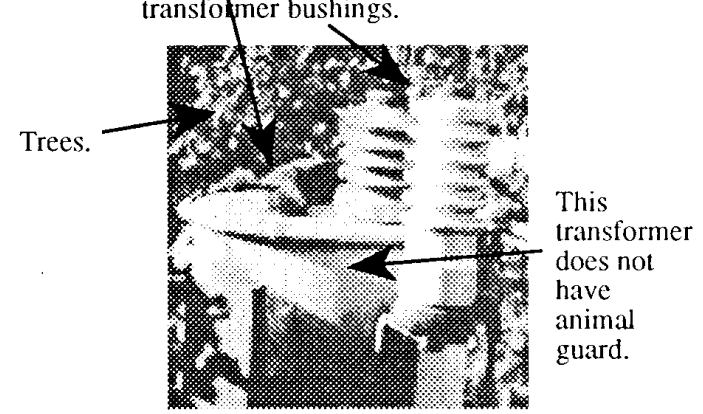

Figure 1. An outage caused by a squirrel on a distribution transformer.

Animals, such as birds, snakes and squirrels, are probably one of the most common causes of distribution faults [8]. Many utility companies have experienced the damages of animal-caused faults, and some have even developed programs to reduce animalcaused faults in an effort to increase the reliability of their distribution systems. In this paper, we will analyze and discuss animal faults based on fault data collected by Duke Power Company. Fig. 2 shows the different categories and percentages of distribution faults experienced by Duke Power between 1987 and 1990. As indicated in this figure, there are three major causes of distribution faults at Duke Power Systems, namely tree faults, animal faults, and Duke Power Equipment failure. The major fault type, tree faults, depends on factors such as the line location and weather conditions. The second major fault type, animal faults, depend on factors such as system configuration and animal behavior. The third major fault type, equipment failure, ranges from lightning arrester failure to capacitor bank failure. Among these three major fault types, animal faults are probably the ones that can be prevented most easily and effectively if appropriate control actions are taken. In the sections that follow, we will focus on the discussion and analysis of animal-caused faults, and then we will present the results of an animal fault prevention technique that can be used effectively to reduce these faults.

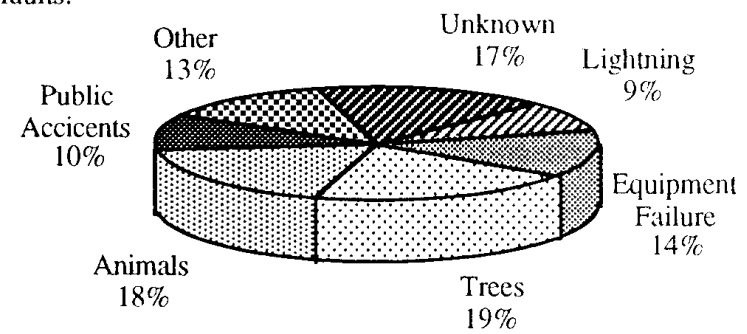

Figure 2. Distribution faults in the Duke Power System (data between 1987 and 1990).

\section{Animal-caused Faults in Distribution Systems}

The occurrences of animal-caused faults are influenced by many factors such as equipment design, system configuration and animal activities. Distribution power equipment manufacturers usually design their equipment based on factors such as efficiency, without much consideration about specific types of faults (except for customized orders) such as squirrels jumps on the transformer - which can cause short circuits. Moreover, since many faults have their own local properties [3], it is unreasonable to expect the manufacturers to design faultless equipment.

The local properties of distribution systems, which include system configurations and animal activities, are illustrated as follows. Consider a distribution system whose clearance between the phase to ground wires in the distribution poles is small. If this system is installed in an area with many active animals, then a large number of animal faults are likely to occur in this system. However, if the active animals were to migrate to other locations due to weather changes or lack of food, then the distribution system will be free of animal faults. But then, if the active animals were to come back due to changes in prey cycles, animal faults in the distribution system will begin to occur again. This phenomenon can be exemplified by Fig. 3, which shows the number of animal fault occurrences and their sites in the Clemson, NC area in 1989 for different seasons. The axis labels on Fig. 3 are the NC (North Carolina) geographic coordinates of the fault occurrences. The number of animal faults, shown as a cross symbol in Fig. 3, varies at different times (season) and different locations. Fig. 3 basically shows that animal-caused faults are a stochastic, time varying process that depend on many different variables.
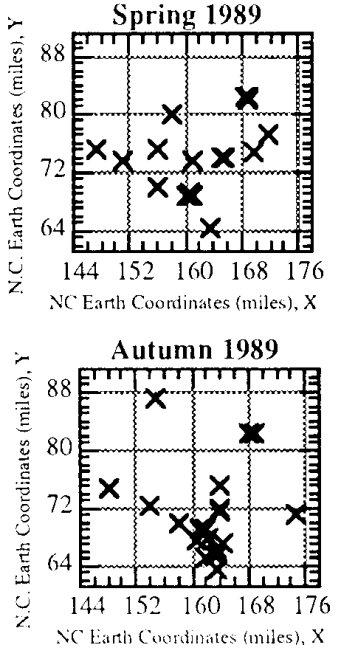

Summer 1989

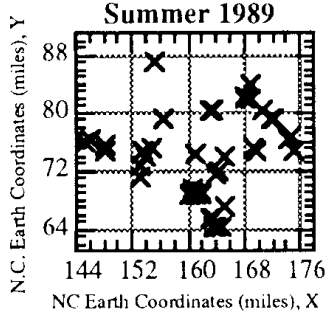

Winter 1989

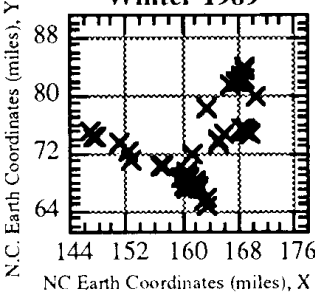

Figure 3. Animal fault locations in the Clemson, SC area for different seasons of 1989.

A better understanding of animal-caused faults is necessary in order to determine if faults that occur in a distribution system are caused by animals and also to take preventive measures to reduce 
these faults in the future. This can be accomplished using a fault investigation perspective. That is, we analyze the animal-caused faults that have occurred, choose and study related factors that determine the causes and effects of animal faults, and then design techniques to eliminate these faults in the future.

\section{Duke Power Fault Data Collection System}

Prior to our discussion about fault data analysis and fault prevention techniques, it may be useful to briefly describe the Duke Power Distribution Fault Data Collection System. In the Duke Power System, distribution faults data are recorded each time a fault current is detected in the distribution system as a result of the operation of a breaker relay, fuse, or other protective device. The recorded data, which includes twenty-two items for each outage, contains information such as the geographical location, circuit ID number, weather condition, and time of the fault occurrence. Some of the information is based on a coding system, designed by Duke Power engineers and summarized in charts, that describes the circumstances under which the outages occurred (e.g., weather condition, season of the year, time of the day). Other information items (e.g., number of phases affected, protective devices used) are based on data collected by equipment that monitor the operation of the distribution system. Duke Power divides the distribution fault causes into 12 categories. Note that in Fig. 2, six of the minor fault categories are grouped into the other category.

Once a permanent fault is detected, crewmen are dispatched to restore or repair the system and to determine the fault causes, if possible. In many cases, some evidence is left when a permanent fault has occurred. For instance, if a transformer fuse is blown and a dead squirrel's body is found near the transformer, this is strong evidence to suggest that the fault was caused by an animal. In this case, crewmen will then input code 4 - animal fault into the database. Another example is a lightning fault which results in a burn mark on the pole or insulation; then the crewman will input code 6-Duke Power Equipment failure (caused by lightning) into the database.

In some cases, evidence indicating what caused the faults may not be found. For example, the dead body of an animal that caused a distribution fault may be picked up by other animals, destroying the evidence for the service men to determine the fault cause. In such a case, the crewman will then enter code $11-$ unknown reason as the cause of the fault. With better understanding of the fault causes in the distribution area, the size of the unknown reason category can be significantly reduced Code 04 , which is the animal-caused faults category, will be investigated in detail in this paper.

Seven regions of the Duke Power System were chosen for detailed fault analysis : Chapel Hill (CHPL), Clemson (CLEM), Durham (DURH), Greenville (GREE), Hickory (HICK), Lancaster (LANC) and Winston-Salem (WINS). These chosen regions are reasonable representations of Duke Power's eastern region, central region, and mountain area; these regions also cover metropolitan areas, cities, small towns, rural areas and wooded areas; new systems and old systems; different types of customers, etc. The animal-caused fault data between 1987 and
1990 (a well represented data set) for these seven regions are used in our study and investigation.

\section{Analysis of Animal-Caused Fault at Duke Power Distribution Systems}

Based on the data available from the chosen seven regions, animal faults are investigated with respect to six factors : weather condition, season, day of the week, time of the day, number of phases affected, and protection device activated. Among the factors under consideration, the weather condition, season, day and time are grouped as influential factors of animal faults, while the number of phases affected and the protection device are grouped as consequence factors, i.e., results of animal faults (Table I).

Table I. Influential and consequence factors of animal faults.

\begin{tabular}{|l||l|}
\hline Influential factors & Consequence factors \\
\hline \hline weather condition & \# of phase affected \\
\hline season of the year & protection device activated \\
\hline day of the week & \\
\hline time of the day & \\
\hline
\end{tabular}

Four different measures have been proposed in [3] for the analysis of distribution faults : (1) actual number of faults, (2) normalized number of faults, (3) relative number of faults, and (4) likelihood of faults. We will use the first three measures in this paper for animal fault analysis. The first measure, actual number of animal faults, is simply the number of animal faults recorded in a particular region. But, in order to reduce the effects of regional factors such as circuit size, the second measure, normalized measure [3,5], can be used :

$$
N_{a}=\frac{\# \text { of animal faults in region } R}{\# \text { of total faults in region } R}(\mathrm{pu}) .
$$

The third measure is the relative measure of animal faults of event $x$ that belong to factor $y$ :

$$
R_{a, x}=\frac{\# \text { of animal faults with event } x \in y \text { in region } R}{\text { \# of total faults } \in y \text { in region } R} \text { (pu).(2) }
$$

In this paper, $y \in$ (weather condition, season, day of the week, time of the day, number of phases affected, protection device), and events for $y=$ weather condition are $x \in$ \{fair, cold, snowy, ..., icy\}, etc. The measure in Eq. (2) gives the relative frequency of event $x$ that belongs to factor $y$ and provides more detailed information for fault analysis of each factor being considered in this paper.

The actual animal fault events of the different regions are displayed in Fig. 4a, with the corresponding normalized values displayed in Fig. 4 b. As we can see from these figures, the total number of animal-caused faults and the normalized number of animal-caused faults vary from region to region. If a particular region has more animals, then the number of animal-caused faults is likely to increase. If a circuit does not have good protection devices against animals, then the relative number of animal faults is also likely to increase. 


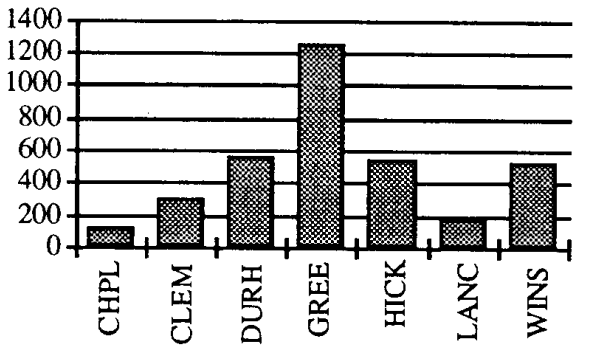

Figure $4 \mathbf{a}$ : Actual number of animal faults in different regions between years $1989-90$

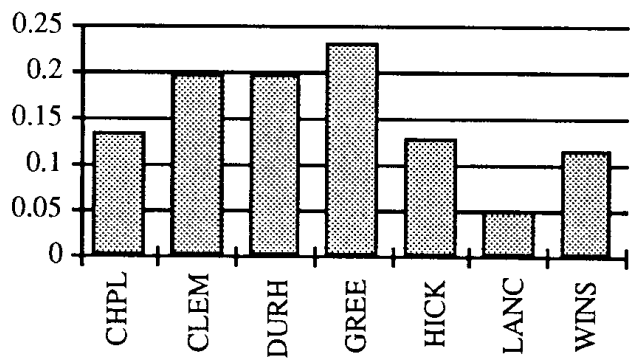

Figure $4 \mathrm{~b}$ : Normalized number of animal faults in different regions between years 1989-90.

For a more detailed animal fault analysis, the normalized animal fault data is further categorized according to weather condition, season of the year, day of the week, time of the day, number of phases affected, and protection devices activated (Figs. $5 \mathrm{a}, \mathrm{b}, \mathrm{c}, \mathrm{d}, \mathrm{e}, \mathrm{f}$ respectively). Figs. 5 are the basis for calculating the relative measures $R_{a x}$ of the animal faults under study.

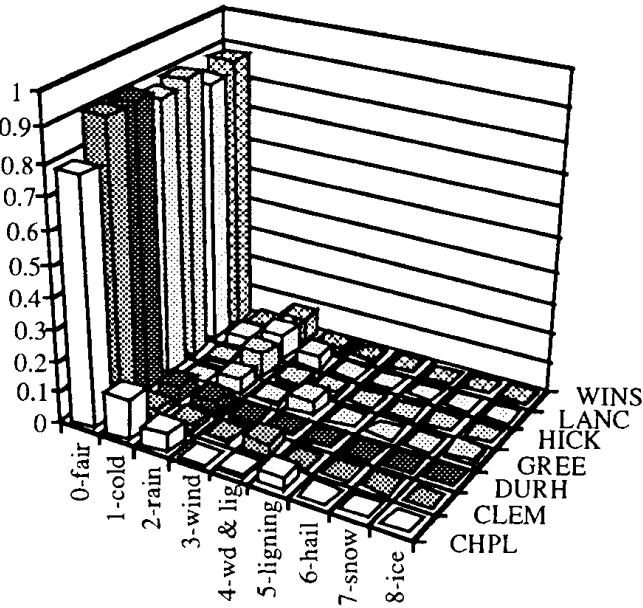

Figure 5a: Relative animal-fault data with respect to weather in different regions.

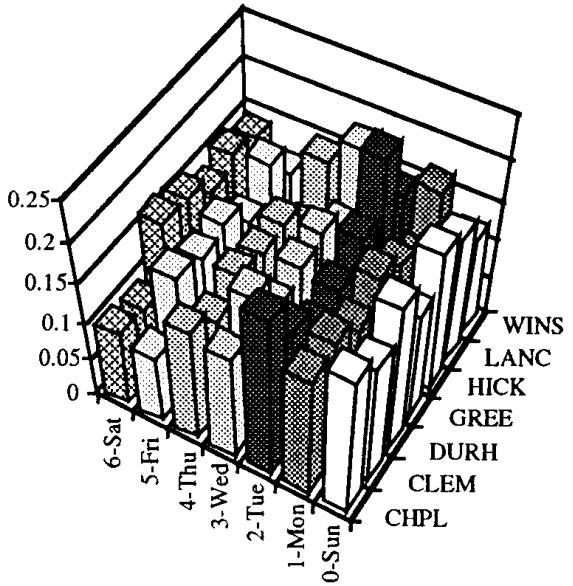

Figure 5b : Relative animal-fault data with respect to day in

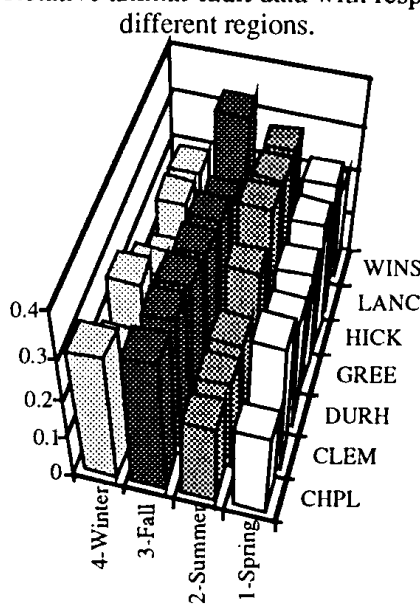

Figure $5 c$ : Relative animal-fault data with respect to season in

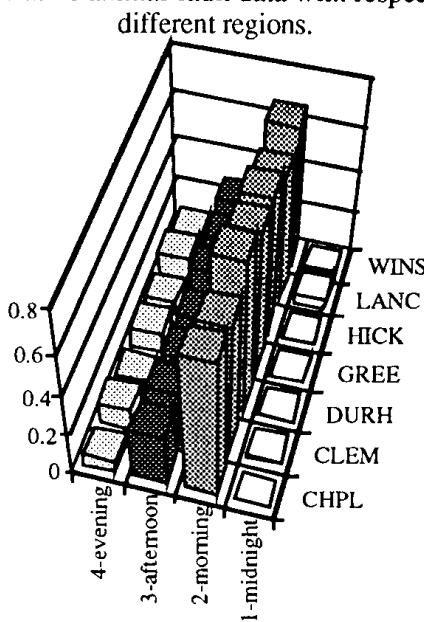

Figure 5d : Relative animal- fault data with respect to time in different regions. 


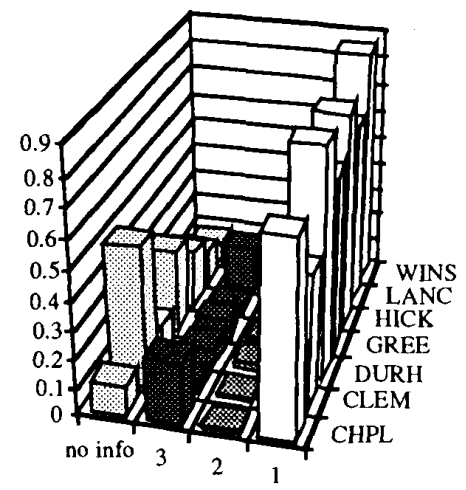

Figure 5e : Relative animal- fault data with respect to number of phases affected in different regions.

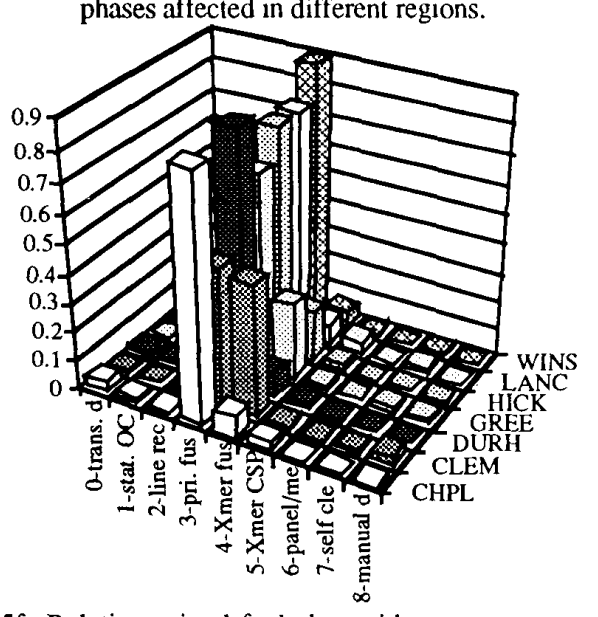

Figure $5 f$ : Relative animal-fault data with respect to protection devices activated in different regions.

Fig. 5a clearly indicates that animal faults more often occur during fair weather conditions. This is intuitively correct because in the Duke Power territory animals tend to be more active in fair weather compared to other weather conditions, such as stormy or snowy weather. Squirrels, which are the primary type of animals that induce animal-caused faults in the Duke Power Systems, are more active in the morning when searching for food - from observation. As a result, there are more animal-caused faults in the morning in the entire Duke Power System than during other times of the day, as shown in Fig. 5d. The animal-caused faults are scarce at midnight because squirrels sleep at night and seldom move around power lines. While no general conclusion can be drawn for animal faults with respect to season and day of week for the Duke Power System, the authors speculate that utility companies in the northern regions may experience less relative animal faults in the winter (with cold winds and perhaps snow) compared to other seasons of the year. Also, as shown in Fig. $5 e$, animal-caused faults usually cause single-phase distribution faults. This is a reasonable conclusion because distribution circuits have more single phase transformers than three phase transformers. Furthermore, even on three phase transformers, it would be extremely difficult for an animal to create a fault involving multiple phases to ground.

As shown in the analysis above, animal faults usually occur in the morning and during fair weather. They usually affect single-phase systems and are likely to blow out the primary or the transformer fuse. We describe in the next section a technique, using animal guards, that can be used to prevent the common animal faults.

\section{An Animal-Fault Prevention Technique}

Many sites of animal caused faults have been investigated on the Duke Power system. These investigations have shown that almost all animal outages could have been prevented if designs had provided adequate clearance between uninsulated conductors and grounded surfaces or between uninsulated phase wires. In some cases, adequate clearances cannot be obtained because of physical or economic constraints. Therefore, an alternative solution is to provide a barrier (usually an animal guard) or to use insulation that has a momentary voltage withstand rating (e.g., insulated transformer lead wire).

Transformer fusing designs have also had an effect on the number of customers affected by animal outages. Until recent years, Duke Power used the Completely Self-Protected (CSP) transformer without any kind of backup fuse. If conventional transformers had been used, then the local external transformer fuse would have operated for many animal caused faults. On the Duke Power system, with the CSP transformer, more tap line fuses were blown, affecting more customers. This difference can be seen in Fig. $5 f$ in relation to the Clemson (CLEM) region. This region was a test site for the CPT transformer (Completely Protected Transformer), which, like the conventional transformer, has a local external fuse for each transformer. Versus the other regions, there is a more even balance between transformer fuse outages and primary line fuse outage. However, local external transformer fusing does not actually prevent animal faults. Furthermore, it may be desirable to prevent these faults, since prevention saves outages for the customer and restoration costs for the utility. If animal fault prevention is necessary, then it can be accomplished by providing adequate clearances, or animal barriers, or adequate insulation, or some combination.

In this section, we will present one of the common techniques to demonstrate the effectiveness of the Control Action Fault Prevention Program. Animals such as squirrels and birds have the tendency to climb up to the transformer on the distribution poles. A transformer fault is usually caused by the insufficient clearance between the phase wire and the transformer case which is connected to the ground. If the clearance is not large enough for animals, and an animal stands on the transformer and touches the phase wire, this will cause a short circuit between the phase to ground wires of the transformer case through the animal body. If the short circuit duration is momentarily, then it will cause a temporary fault that may result in an instantaneous flickering. Electrical distribution structures must have adequate clearances between phases and between phases and grounded surfaces. If air clearance cannot be provided in a 
practical manner, then insulated coverings must be installed to prevent animal outages.

The flock of birds shown in Fig. 6 can give a severe test to any structure. If the short circuit duration is long enough to trigger one of the protection devices, such as the transformer fuse, then it will cause a permanent fault. In either case, if the animal is killed (electrocuted) and the dead body is found near the site, then it is a strong evidence to suggest that an animal caused the fault. If the animal was only hurt and ran away or the dead body could not be found, then we can use different techniques, such as AFI-ANN [4], to identify if the fault was caused by an animal. Actually, in Fig. 6, a bird landed on this structure and shorted out the insulator. This pole does not meet proper clearance specifications; the hardware is grounded and there is not enough distance across the insulator to prevent an outage. The fault resulted in a station breaker operation. The investigation team found the fault location using a device designed to measure the electrical parameters of the fault. The approximate location of the fault from the substation was determined, and the probable fault location area was searched.

This flock of birds can easily cause outages on this distribution pole structure.

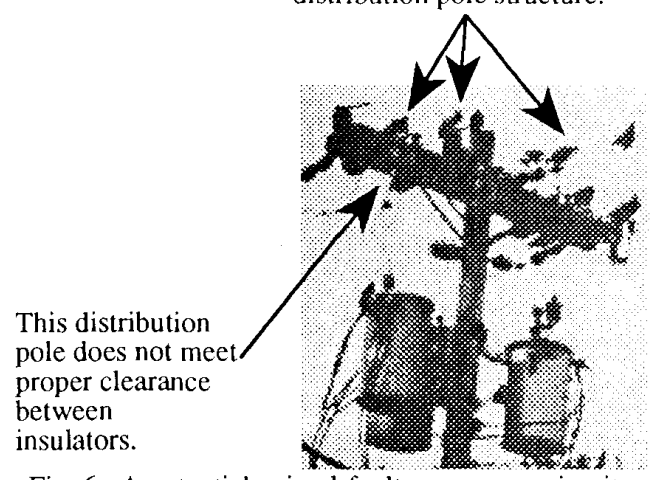

Fig. 6. A potential animal-fault occurrence circuit.

An animal guard, shown in Fig. 7, can be used to easily solve the animal faults problem. Once a circuit is identified as one frequented by active animals, it will be worthwhile to install an animal guard on the bushing of the transformer. Animal guards are inexpensive and can effectively prevent animal faults from occurring by protecting from the short circuit caused by an animal standing between the phase wire and the transformer since the location on which the animal stands is insulated. Table II shows the number of animal faults before and after the installation of animal guards on a Duke Power circuit that in the past frequently suffered from animal-caused faults. As shown in Table II, the circuit had many animal-caused faults prior to 1989. At the end of 1988, the distribution transformers of the circuit were installed with animal guards. The reliability indices used in Table II for sustained distribution faults are Frequency Index (SAIFI) [9] :

$$
\text { SAIFI }=\frac{\text { Customers Interrupted }}{\text { Customers Served }},
$$

which indicates how many times per year the average customer will be interrupted; Duration Index (CAIDI) :

$$
\text { CAIDI }=\frac{\text { Customer-Minutes }}{\text { Customers Interrupted }}
$$

which indicates the average duration of outages by customers who actually experience an outages; and Internuption-Minutes Per Customer (SAIDI) :

$$
\text { SAIDI }=\frac{\text { Customer-Minutes }}{\text { Customer Served }},
$$

which indicates the average interruption minutes caused by the distribution fault per year.

Among the three indices, as indicated in Eqs. (3) - (5), SAIFI and SAIDI are the indices highly related with the number of faults that occurred in the systems. As shown in Fig. 8, the animal guard installation control action on the circuit has significantly reduced the number of animal-caused faults, as well as the reliability indices SAIFI and SAIDI. (Note that SAIFI index number is multiplied by 10 in order to be seen in the figure.)

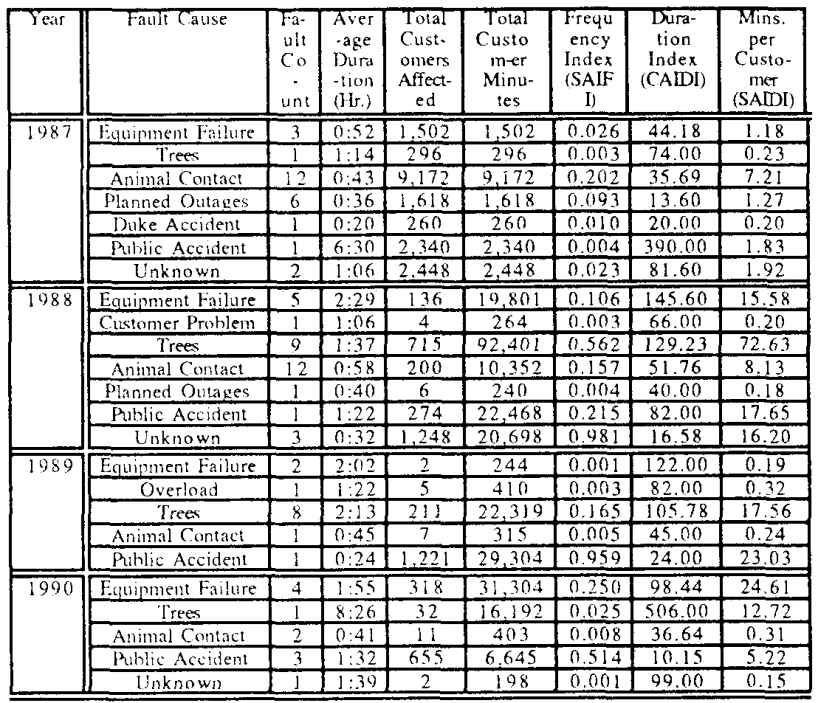

Table II. Animal faults on a Duke Power circuit before and after the installation of animal guards.

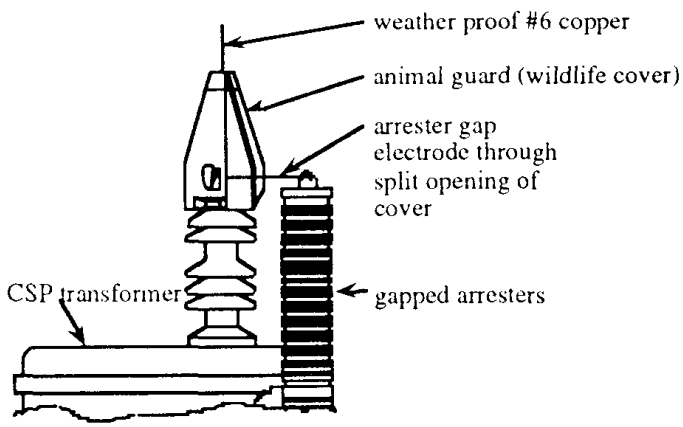

Fig. 7. Picture of an animal guard used to prevent animal-caused distribution faults. 


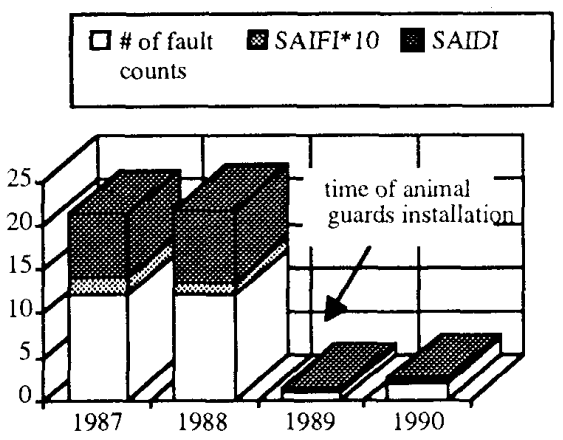

Fig. 8. Animal fault counts and reliability indices of the distribution circuit for the outages caused by animal faults before and after the installation of animal guards on the circuit.

\section{Conclusions}

This paper has discussed the importance of distribution fault prevention in power system operations. Among the distribution faults, animal faults are probably the one that can be prevented most effectively by Control Action Fault Prevention Techniques. In this paper, we have discussed and analyzed animal-caused faults in power distribution systems based on actual data collected by Duke Power Company, by using different measures for analysis to gain a better understanding of the causes and consequences of animal-caused faults. This paper has also discussed about the use of animal guards as an effective action to reduce animal-caused faults in distribution systems. Significant results of this fault prevention technique for distribution systems has also been presented.

\section{Acknowledgment}

The authors of this paper would like to acknowledge the support of Electric Power Research Center project grant 5-31294. Duke Power Company project grant 5-30550, and National Science Foundation Grant ECS-9311833.

\section{Reference}

[1] J.G. Anderson, P.P. Barker, I.S. Grant, D.J. Lawrence, Characteristics of Lightning Surges on Distribution Lines, EPRI EL-6782, Research Project 2542-1, Interim Report, May 1990.

[2] J.J. Burke, D.A. Douglass, D.J. Lawrence, Distribution Fault Current Analysis, EPRI EL-3085, Research Project 1209-1, Final Report, May 1983.

[3] Mo-yuen Chow, Leroy S. Taylor, "A Novel Approach for Distribution Fault Analysis," Presented in PES/WM'93. Columbus, Ohio, 1/31/93 - 2/4/93, to be published in IEEE Transaction on Power Delivery, 1993.

[4] Mo-yuen Chow, Sui Oi Yee, Leroy S. Taylor, "Recognizing Animal-Caused Faults in Power Distribution Systems Using Artificial Neural Networks," presented in PES SM, 1992, Seattle, WA: to be published in IEEE Transactions on Power Delivery, 1993.

[5] E. R. Dougherty, Probability and Statistics for the Engineering. Computing, and Physical Sciences, Prentice Hall, 1990.
[6] IEEE Tutorial Text, Detection of Downed Conductors on Utility Distribution Systems, Course Text 90EH0310-3PWR.

[7] C.J. Kim, B. Don Russel, "A Learning Method for Use in Intelligent Computer Relays for High Impedance Faults," IEEE Transactions on Power Delivery, 1991, Vol. 6, No. 1. pp. 109-115

[8] Leroy S. Taylor, "Finding the Exact Cause of Fault Currents," Duke Power Company Technical Report, February, 1988.

[9] Leroy S. Taylor, Vance Martin, System Reliability = Training and Reference Manual, Duke Power Company Manual, November 1992.

[10] Cheryl M. Warren, "The Effect of Reducing Momentary Outages on Distribution Reliability Indices," T\&D Exposition, Dallas, TX, September, 1991.

\section{Biography}

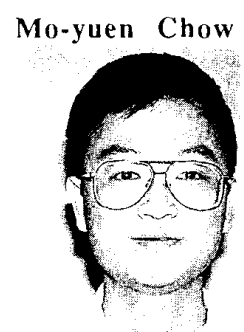

Mo-yuen Chow earned the B.S. degree at University of Wisconsin-Madison (June 1982), M. Eng. degree at Cornell University (August 1983), and Ph.D. degree at Cornell University (August 1987), all the degrees are in Electrical Engineering. After graduation, he joined the faculty of North Carolina State University. Currently, Dr. Chow is an Associate Professor in the Electrical and Computer Engineering Department at North Carolina State University. His research interests are System Monitoring Fault Detection and Control, Artificial Neural Networks and Fuzzy Logic.

\section{Leroy S. Taylor}

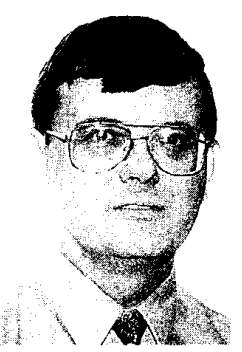

Leroy S. Taylor is a Senior Distribution Engineer for Duke Power Company, and is a registered professional engineer in North Carolina. He was born in 1949 in Greenville, N.C. and received a B.A. degree (Physics) from the University of North Carolina in 1971. Joining Duke Power in 1977, he acquired extensive experience in distribution system engineering, operation, and construction. Since 1987 he has conducted intensive investigations on the cause of power quality disturbances which originate in the distribution system. He has also redesigned several Duke Power mainframe reporting systems used to evaluate and improve distribution system reliability and power quality. 